\title{
A nonlocal connection between certain linear and nonlinear ordinary differential equations/oscillators
}

\author{
V. K. Chandrasekar ${ }^{\dagger}$, M. Senthilvelan ${ }^{\dagger}$, Anjan Kundu ${ }^{\dagger \dagger}$ and \\ M. Lakshmanan ${ }^{\dagger}$ \\ ${ }^{\dagger}$ Centre for Nonlinear Dynamics, Department of Physics, Bharathidasan University, \\ Tiruchirappalli - 620 024, India \\ ${ }^{\dagger}$ Saha Institute of Nuclear Physics, Sector 1, Block AF, Bidhan Nagar, Kolkata - 700 \\ 064, India
}

\begin{abstract}
We explore a nonlocal connection between certain linear and nonlinear ordinary differential equations (ODEs), representing physically important oscillator systems, and identify a class of integrable nonlinear ODEs of any order. We also devise a method to derive explicit general solutions of the nonlinear ODEs. Interestingly, many well known integrable models can be accommodated into our scheme and our procedure thereby provides further understanding of these models.
\end{abstract}

\section{Introduction}

In a recent paper, we have shown that the modified Emden type equation with additional linear forcing,

$$
\ddot{x}+3 k x \dot{x}+k^{2} x^{3}+\lambda x=0,
$$

where over dot denotes differentiation with respect to $t$ and $k$ and $\lambda$ are arbitrary parameters, exhibits certain unusual nonlinear dynamical properties [1]. For a particular sign of the control parameter, namely, $\lambda>0$, the frequency of oscillations of the nonlinear oscillator (11) is completely independent of amplitude and remains the same as that of the linear harmonic oscillator, thereby showing that the amplitude dependence of frequency is not necessarily a fundamental property of nonlinear dynamical phenomena [1]. In this case, namely, $\lambda>0$, the system admits the explicit sinusoidal periodic solution

$$
x(t)=\frac{A \sin (\omega t+\delta)}{1-\left(\frac{k}{\omega}\right) A \cos (\omega t+\delta)}, \quad 0 \leq A<\frac{\omega}{k}, \quad \omega=\sqrt{\lambda},
$$

where $A$ and $\delta$ are arbitrary constants. The system (1D) exhibits certain other unusual properties also. For more details on the dynamics of this equation one may refer to [1].

In the same paper, we have also noted that the solution (2) can be derived unambiguously by introducing a nonlocal transformation,

$$
U=x e^{k \int_{0}^{t} x\left(t^{\prime}\right) d t^{\prime}}
$$


and transforming equation (11) to the linear harmonic oscillator equation,

$$
\ddot{U}+\lambda U=0 \text {. }
$$

For $\lambda>0$, the solution is $U=A \sin (\omega t+\delta)$, where $A$ and $\delta$ are arbitrary constants and the frequency, $\omega=\sqrt{\lambda}$, is independent of the amplitude. Using (3) one finds that

$$
\dot{x}=\frac{\dot{U}}{U} x-k x^{2} .
$$

Integrating Riccati type equation (5) and absorbing the integration constant with the existing ones, one can obtain the solution (2). We mention here that the solution (2) can be derived in a number of ways, for more details one may again refer to [1] and [2]. One may also note that in the case $\lambda=0$ the transformation (3) modifies equation (11) to the free particle equation. The linearization and integrability properties of equation (11) with $\lambda=0$ has received a considerable attention in the nonlinear dynamics literature, see for example, Refs. [3-6].

Now the question that naturally arises is whether the nonlocal transformation which connects the linear and nonlinear oscillators, namely, Eqs. (11) and (4) is a rare one or there exists a wider class of nonlinear dynamical systems that are connected with linear oscillators in a hidden way? Our studies reveal that there exists a class of linear oscillators that are connected with nonlinear oscillator equations through nonlocal transformations. For example, let us consider a damped linear harmonic oscillator, instead of the undamped linear harmonic oscillator, that is,

$$
\ddot{U}+c_{1} \dot{U}+c_{2} U=0,
$$

where $c_{1}$ and $c_{2}$ are arbitrary parameters, and consider a more general form of nonlocal transformation, that is,

$$
U=x(t) e^{\int_{0}^{t} f\left(x\left(t^{\prime}\right)\right) d t^{\prime}},
$$

where $f(x(t))$ is an arbitrary function of $x$. Now substituting (7) into (6) we get a nonlinear ODE of the form

$$
\ddot{x}+\left(2 f+x f^{\prime}+c_{1}\right) \dot{x}+\left(f^{2}+c_{1} f+c_{2}\right) x=0,
$$

where prime denotes differentiation with respect to $x$. Interestingly, one can now see that for certain specific forms of the function $f$, the associated nonlinear ODEs become the well known and well studied models in the current nonlinear dynamics literature. To cite a few, first let us consider the case $f(x)=k x$ in (77). The associated nonlinear ODE,

$$
\ddot{x}+\left(c_{1}+3 k x\right) \dot{x}+k^{2} x^{3}+c_{1} k x^{2}+c_{2} x=0,
$$

is nothing but the generalized modified Emden type equation (MEE) whose integrability properties have been studied in detail in Refs. [3-10]. It is one of the linearizable second order nonlinear ODEs which admits eight parameter Lie point symmetries [4].

Choosing $f(x)=k x^{2}$ in (7), one gets the generalized force-free Duffing-van der Pol oscillator (DVP) equation

$$
\ddot{x}+\left(c_{1}+4 k x^{2}\right) \dot{x}+k^{2} x^{5}+k c_{1} x^{3}+c_{2} x=0,
$$


as its nonlinear counterpart. More details on the mathematical aspects and the underlying dynamics of this equation can be found in Refs. [9-13]. On the other hand fixing $f(x)=\frac{k}{x^{2}}$, one gets another integrable nonlinear second order ODE of the form [9]

$$
\ddot{x}+c_{1} \dot{x}+c_{2} x+\frac{k c_{1}}{x}+\frac{k^{2}}{x^{3}}=0 .
$$

The examples illustrated above clearly demonstrate that one can systematically classify a class of integrable nonlinear ODEs through this way.

Motivated by these observations, in this paper, we carry out a detailed investigation

on the nonlocal connection between certain linear ODEs and their nonlinear counterparts of any order. To make our study a systematic one we start our investigations at the level of second order and end with $n$th order equations. Besides constructing integrable nonlinear equations we also point out the importance of these nonlinear equations and obtain their solutions and thereby bring out the significance of these systems.

The plan of the paper is as follows. In Sec. 2, we make a detailed study on the nonlocal connection between damped linear harmonic oscillator equation and certain nonlinear oscillator equations of second order and describe a method of deriving general solutions of the nonlinear ODEs. We extend this procedure to third and $n$th order ODEs in Secs. 3 and 4, respectively. We present a general theory which connects the variable coefficient linear ODEs with nonlinear ODEs and the method of finding the general solution in Sec. 5. We present our conclusion in Sec. 6.

\section{Second order ODEs}

To begin with let us consider a linear second order ODE of the form (66), whose general solution is $U=a(t)$, where $a(t)$ is a known function. Now we consider a nonlocal transformation of the form

$$
U=x^{n} e^{\int_{0}^{t}\left(\beta\left(t^{\prime}\right) x^{m}+\gamma\left(t^{\prime}\right)\right) d t^{\prime}},
$$

where $n$ and $m$ are constants and $\beta(t)$ and $\gamma(t)$ are arbitrary functions of $t$, and substitute it into (6) so that the latter becomes a nonlinear second order ODE of the form

$$
\ddot{x}+(n-1) \frac{\dot{x}^{2}}{x}+\frac{\beta^{2}}{n} x^{2 m+1}+b_{1}(t, x) \dot{x}+b_{2}(t) x^{m+1}+b_{3}(t) x=0,
$$

where

$$
\begin{aligned}
& b_{1}(t, x)=\frac{1}{n}\left(2 n \gamma+n c_{1}+(m+2 n) \beta x^{m}\right), \\
& b_{2}(t)=\frac{1}{n}\left(\dot{\beta}+2 \gamma \beta+c_{1} \beta\right), \\
& b_{3}(t)=\frac{1}{n}\left(\dot{\gamma}+\gamma^{2}+\gamma c_{1}+c_{2}\right) .
\end{aligned}
$$

Obviously the transformation (12) corresponds to the special case, $f(x)=$ $\beta(t) x^{m}, \gamma(t)=0$, in the transformation (7) but with the prefactor of the exponential function taken as $x^{n}$, where $n$ is arbitrary. We have been motivated to choose this form due to the examples illustrated in the introduction. In all these cases, one might have 
noted that the function $f(x)$ is a simple polynomial one. As a consequence, we would like to generalize this form and see the outcome. Such a generalization is indicated in Sec. 5. However, here we note that the form (13) itself yields several important nonlinear ODEs as see below.

Now the question is how to construct the general solution for the nonlinear equation (13) from the known solution of (6). This can be done by using the identity,

$$
\frac{\dot{U}}{U}=\frac{n \dot{x}}{x}+\beta(t) x^{m}+\gamma(t)
$$

derived from equation (12). Since $U=a(t)$, where $a(t)$ is a known function, the solution of the second order linear ODE (6), equation (15) can be brought to the form

$$
\dot{x}=(\hat{a}(t)-\gamma(t)) \frac{x}{n}-\frac{\beta(t)}{n} x^{m+1},
$$

where $\hat{a}=\frac{\dot{a}}{a}$. Solving equation (16) we get the general solution for the equation (13) in the form

$$
x(t)=e^{\frac{1}{n} \int_{0}^{t}\left(\hat{a}\left(t^{\prime}\right)-\gamma\left(t^{\prime}\right)\right) d t^{\prime}}\left[C+\frac{m}{n} \int_{0}^{t}\left(\beta\left(t^{\prime}\right) e^{\frac{m}{n} \int_{0}^{t^{\prime}}\left(\hat{a}\left(t^{\prime \prime}\right)-\gamma\left(t^{\prime \prime}\right)\right) d t^{\prime \prime}}\right) d t^{\prime}\right]^{\frac{-1}{m}} .
$$

Note that the equation (17) contains three arbitrary constants (two in $\hat{a}(t)$, since $a(t)$ is a general solution of the second order equation (6), and another constant $C$ ). However, one can absorb one arbitrary constant with the other two and rewrite the solution in such a way that it contains only two arbitrary constants, as we see below.

Let us assume that the solution of (6) be written in the form

$$
U(t)=a(t)=I_{1} e^{m_{1} t}+I_{2} e^{m_{2} t},
$$

where $I_{i}$ 's, $i=1,2$, are integration constants and $m_{i}$ 's, $i=1,2$, are the roots of the auxiliary equation associated with the ODE (6). From (18) we get

$$
\hat{a}(t)=\frac{\dot{a}}{a}=\frac{\dot{U}}{U}=\frac{m_{1} \hat{I}_{1} e^{m_{1} t}+m_{2} e^{m_{2} t}}{\hat{I}_{1} e^{m_{1} t}+e^{m_{2} t}},
$$

where $\hat{I}_{1}=\frac{I_{1}}{I_{2}}$. Integrating both sides of the equation (119) we get

$$
\int_{0}^{t} \hat{a}\left(t^{\prime}\right) d t^{\prime}=\log \left(\hat{I}_{1} e^{m_{1} t}+e^{m_{2} t}\right)=\log \left(\frac{a(t)}{I_{2}}\right) .
$$

Substituting the expression (20) into equation (17), we get a general solution for the equation (13) in the form

$$
\begin{aligned}
x(t)=\left(\frac{a(t)}{I_{2}}\right)^{\frac{1}{n}} & e^{\frac{-1}{n} \int_{0}^{t} \gamma\left(t^{\prime}\right) d t^{\prime}} \\
& \times\left[C+\frac{m}{n} \int_{0}^{t}\left(\beta\left(t^{\prime}\right)\left(\frac{a\left(t^{\prime}\right)}{I_{2}}\right)^{\frac{m}{n}} e^{\frac{-m}{n} \int_{0}^{t^{\prime}} \gamma\left(t^{\prime \prime}\right) d t^{\prime \prime}}\right) d t^{\prime}\right]^{\frac{-1}{m}} .
\end{aligned}
$$

Now the general solution (21) contains only two arbitrary constants, namely, $\hat{I}_{1}$ and $C$. In the following, we consider certain important sub-cases of the linear oscillator (6) and discuss their nonlinear counterparts and their physical significance. 


\subsection{Case (i) $c_{1}=0$ and $c_{2}=0$}

In this case the linear equation (6) is nothing but the free particle equation. The associated nonlinear equation can be fixed from (13) by restricting $c_{1}=0$ and $c_{2}=0$. The general solution of the nonlinear equation turns out to be

$$
x(t)=\left(t+I_{1}\right)^{\frac{1}{n}} e^{\frac{-1}{n} \int_{0}^{t} \gamma\left(t^{\prime}\right) d t^{\prime}}\left[C+\frac{m}{n} \int_{0}^{t}\left(\beta\left(t^{\prime}\right)\left(t^{\prime}+I_{1}\right)^{\frac{m}{n}} e^{-\frac{m}{n} \int_{0}^{t^{\prime}} \gamma\left(t^{\prime \prime}\right) d t^{\prime \prime}}\right) d t^{\prime}\right]^{\frac{-1}{m}},
$$

where $I_{1}$ and $C$ are arbitrary constants.

The interesting fact is that equation (13) contains a family of important nonlinear ODEs. To mention one such example let us choose $n=1, \gamma(t)=0$ and $\beta(t)=k$ so that equation (13) now becomes

$$
\ddot{x}+(m+2) k x^{m} \dot{x}+k^{2} x^{2 m+1}=0 .
$$

The invariance, integrability properties and direct linearization through generalized transformation of equation (23) can be found in Refs. [6,9,10]. From our above results, the general solution of (23) can be fixed easily from (22) in the form

$$
x(t)=\left(t+I_{1}\right)\left[C+\frac{m k}{(m+1)}\left(I_{1}+t\right)^{m+1}\right]^{\frac{-1}{m}}
$$

which exactly coincides with the results obtained through other methods $[6,9,10]$.

\subsection{Case (ii) $c_{1}=0$ and $c_{2}=$ constant}

In this case, from equation ([6), we have the linear harmonic oscillator equation at our hand. The nonlocal transformation (12) transforms the linear harmonic oscillator equation, $\ddot{U}+c_{2} U=0$, to the nonlinear ODE (13) with $c_{1}=0$. The general solution of (13) turns out to be

$$
\begin{aligned}
x(t)=[\cos (\omega t+\delta)]^{\frac{1}{n}} e^{\frac{-1}{n} \int_{0}^{t} \gamma\left(t^{\prime}\right) d t^{\prime}} & \\
& \times\left[C+\frac{m}{n} \int_{0}^{t}\left(\beta\left(t^{\prime}\right) e^{-\frac{m}{n} \int_{0}^{t^{\prime}} \gamma\left(t^{\prime \prime}\right) d t^{\prime \prime}} \cos ^{\frac{m}{n}}\left(\omega t^{\prime}+\delta\right)\right) d t^{\prime}\right]^{\frac{-1}{m}},
\end{aligned}
$$

where $\omega=\sqrt{c_{2}}$ and $\delta$ and $C$ are arbitrary constants.

Interestingly, one can deduce certain important nonlinear ODEs from (13) with $c_{1}=0$. One such interesting case is $n=1, m=1, \gamma(t)=0$ and $\beta(t)=k$. In this case we get the equation (11). The unusual dynamical properties exhibited by this equation has already been pointed out in the introduction.

Now we choose $n=1, m=-2, \gamma(t)=0$ and $\beta(t)=k$ in equation (13) so that the latter becomes

$$
\ddot{x}+c_{2} x+\frac{k^{2}}{x^{3}}=0
$$

which is another important nonlinear ODE which arises in different areas of physics and has been studied in detail in Ref. $[14,15]$. The solution can be derived from (25) in the form

$$
x(t)=\cos (\omega t+\delta)\left[C-\frac{2 k}{\omega} \tan (\omega t+\delta)\right]^{\frac{1}{2}}, \omega=\sqrt{c_{2}} .
$$


Note that the solution defines a well defined harmonic periodic oscillation with period $T=\frac{2 \pi}{\sqrt{c_{2}}}$ which is the same as that of the unperturbed simple harmonic oscillator. This nonlinear oscillator again supports our argument that the amplitude dependence of frequency is not necessarily a fundamental property of nonlinear oscillations.

On the other hand fixing $n=1, m=2, \gamma(t)=-k_{2}$ and $\beta(t)=k_{1}$ in equation (13) we get

$$
\ddot{x}+\left(4 k_{1} x^{2}-2 k_{2}\right) \dot{x}+k_{1}^{2} x^{5}-2 k_{1} k_{2} x^{3}+\left(c_{2}+k_{2}^{2}\right) x=0,
$$

which is nothing but the generalized force-free Duffing-van der Pol nonlinear oscillator equation $[9,11-13]$. The general solution can again be fixed from (25) as

$$
\begin{aligned}
x(t) & =\cos (\omega t+\delta) \\
& \times\left[C e^{-2 k_{2} t}+\frac{k_{1}}{2 k_{2}}+\frac{k_{1}}{2\left(\omega^{2}+k_{2}^{2}\right)}\left(k_{2} \cos 2(\omega t+\delta)+\omega \sin 2(\omega t+\delta)\right)\right]^{-\frac{1}{2}}
\end{aligned}
$$

which exactly coincides with the result obtained through other methods $[9,11-13]$.

Finally, let us consider the case $n=1, m=q, \gamma(t)=-k_{2}$ and $\beta(t)=k_{1}$ in equation (13). In this case we end up with the following nonlinear ODE

$$
\ddot{x}+\left((q+2) k_{1} x^{q}-2 k_{2}\right) \dot{x}+\left[\left(k_{1} x^{q}-k_{2}\right)^{2}+c_{2}\right] x=0 .
$$

Equation (30) admits the following general solution

$$
x(t)=\cos (\omega t+\delta) e^{k_{2} t}\left[C+q k_{1} \int_{0}^{t}\left(e^{q k_{2} t^{\prime}} \cos ^{q}\left(\omega t^{\prime}+\delta\right)\right) d t^{\prime}\right]^{-\frac{1}{q}} .
$$

We mention here that for even values of $q$ and positive values of $k_{1}$ and $k_{2}$ equation (30) exhibits limit cycle oscillations [12].

\subsection{Case (iii) $c_{1}, c_{2}=$ constant}

In this case the nonlocal transformation connects the damped harmonic oscillator ODE with the nonlinear ODE (13). From (21), the general solution of equation (13) with $c_{1}$ and $c_{2}$ taking constant values turns out to be

$$
\begin{aligned}
x(t)=\left(e^{\left(\frac{-c_{1}+\omega}{2}\right) t}+I_{1} e^{-\left(\frac{c_{1}+\omega}{2}\right) t}\right)^{\frac{1}{n}} e^{\frac{-1}{n} \int_{0}^{t} \gamma\left(t^{\prime}\right) d t^{\prime}} & \\
& \times\left[C+\frac{m}{n} \int_{0}^{t}\left(\beta\left(t^{\prime}\right)\left(\frac{I_{1}+e^{\omega t^{\prime}}}{e^{\left(\frac{c_{1}+\omega}{2}\right) t^{\prime}}}\right)^{\frac{m}{n}} e^{-\frac{m}{n} \int_{0}^{t^{\prime}} \gamma\left(t^{\prime \prime}\right) d t^{\prime \prime}}\right) d t^{\prime}\right]^{\frac{-1}{m}},
\end{aligned}
$$

where $\omega=\sqrt{c_{1}^{2}-4 c_{2}}$ and $I_{1}$ and $C$ are arbitrary constants.

In this case also one can identify several physically interesting nonlinear oscillator equations. For example, choosing $n=1, m=1, \gamma(t)=0$ and $\beta(t)=k$ in equation (13) we get an equation of the form (91). The general solution can be written from (32) in the form

$$
x(t)=\left(\frac{2 c_{2}\left(I_{1}+e^{\omega t}\right)}{C e^{\left(\frac{c_{1}+\omega}{2}\right) t}-k\left(c_{1}-\omega\right) I_{1}-k\left(c_{1}+\omega\right) e^{\omega t}}\right),
$$

where $\omega=\sqrt{c_{1}^{2}-4 c_{2}}$. Indeed our result exactly coincides with the result obtained in the Refs. [8,9]. 
The choice $n=1, m=2, \gamma(t)=0$ and $\beta(t)=k$ leads us to an equation of the form (10). The general solution of (10) can be fixed easily from (32) in the form

$$
x(t)=\left(\frac{2 c_{1} c_{2}\left(I_{1}+e^{\omega t}\right)^{2}}{C e^{\left(\frac{c_{1}+\omega}{2}\right) t}-k I_{1}^{2} c_{1}\left(c_{1}-\omega\right)-k c_{1}\left(c_{1}+\omega\right) e^{2 \omega t}-8 k I_{1} c_{2} e^{\omega t}}\right)^{\frac{1}{2}},
$$

which indeed the general solution of the genaralized force-free DVP oscillator equation [9].

In this section, besides the general case, we considered three different linear equations, namely, free particle equation, linear harmonic oscillator and damped linear harmonic equations and demonstrated how they are related to the well known nonlinear models through nonlocal transformations. In the following section, we extend the theory to third order ODEs and study the outcome.

\section{Third order ODEs}

Let us consider a linear third order ODE of the form

$$
\dddot{U}+c_{1} \ddot{U}+c_{2} \dot{U}+c_{3} U=0,
$$

where $c_{1}, c_{2}$ and $c_{3}$ are arbitrary constants. The nonlocal transformation (12) transforms (35) to the nonlinear ODE of the form

$$
\begin{aligned}
\dddot{x}+[3(n-1) & \left.\frac{\dot{x}}{x}+d_{1}(t, x)\right] \ddot{x}+(n-1)(n-2) \frac{\dot{x}^{3}}{x^{2}}+d_{2}(t, x) \dot{x}^{2} \\
& +d_{3}(t, x) \dot{x}+\frac{\beta^{3}}{n} x^{3 m+1}+d_{4}(t) x^{2 m+1}+d_{5}(t) x^{m+1}+d_{6}(t) x=0,
\end{aligned}
$$

where

$$
\begin{aligned}
& d_{1}(t, x)=\frac{1}{n}\left(3 n \gamma+n c_{1}+\beta(m+3 n) x^{m}\right), \\
& d_{2}(t, x)=\frac{1}{n}\left((m(m+2 n)+(n-1)(m+3 n)) \beta x^{m-1}+n(n-1)\left(3 \gamma+c_{3}\right) x^{-1}\right), \\
& d_{3}(t, x)=\frac{1}{n}\left(\left(3 \gamma \beta(m+2 n)+\dot{\beta}(2 m+3 n)+c_{1}(m+2 n) \beta\right) x^{m}\right. \\
& \left.\quad+3 \beta^{2}(m+n) x^{2 m}+n\left(3 \dot{\gamma}+3 \gamma^{2}+2 c_{1} \gamma+c_{2}\right)\right), \\
& d_{4}(t)=\frac{1}{n}\left(3 \beta \dot{\beta}+3 \gamma \beta^{2}+\beta^{2} c_{1}\right), \\
& d_{5}(t)=\frac{1}{n}\left(\ddot{\beta}+3(\dot{\gamma} \beta+\gamma \dot{\beta})+3 \beta \gamma^{2}+\left(c_{1} \dot{\beta}+c_{2} \beta\right)+2 c_{1} \gamma \beta\right), \\
& d_{6}(t)=\frac{1}{n}\left(\ddot{\gamma}+3 \gamma \dot{\gamma}+\gamma^{3}+c_{1}\left(\dot{\gamma}+\gamma^{2}\right)+\gamma c_{2}+c_{3}\right) .
\end{aligned}
$$

To obtain the exact solution, $x(t)$, of equation (36) we again apply the results of Eqs. (15)-(17) to the present case. Since we are using the same form of nonlocal transformation (12) to transform the nonlinear ODE (36) to the linear ODE (35), the general solution of (36) looks exactly the same form as (17). The only difference is that the general solution, in the present case, contains four integration constants (three of 
them come out from the solution of the linear ODE (35) and the remaining one from solving (16) $)$. Now let us rewrite the general solution in such a way that it contains only three arbitrary constants by absorbing the fourth one. To do so let us follow the same procedure which we adopted in the case of second order ODEs.

Let us write the general solution of equation (35) in the form

$$
U(t)=a(t)=I_{1} e^{m_{1} t}+I_{2} e^{m_{2} t}+I_{3} e^{m_{3} t},
$$

where $I_{i}$ 's, $i=1,2,3$, are integration constants and $m_{i}$ 's, $i=1,2,3$, are the roots of the auxiliary equation associated with the differential equation (35). From (38) we get

$$
\hat{a}(t)=\frac{\dot{a}}{a}=\frac{\dot{U}}{U}=\frac{m_{1} \hat{I}_{1} e^{m_{1} t}+m_{2} \hat{I}_{2} e^{m_{2} t}+m_{3} e^{m_{3} t}}{\hat{I}_{1} e^{m_{1} t}+\hat{I}_{2} e^{m_{2} t}+e^{m_{3} t}},
$$

where $\hat{I}_{i}=\frac{I_{i}}{I_{3}}, i=1,2$. Integrating both sides of equation (39) we get

$$
\int_{0}^{t} \hat{a}\left(t^{\prime}\right) d t^{\prime}=\log \left(\hat{I}_{1} e^{m_{1} t}+\hat{I}_{2} e^{m_{2} t}+e^{m_{3} t}\right)=\log \left(\frac{a(t)}{I_{3}}\right) .
$$

Substituting the expression (40) into (17), we get the general solution for the equation (36) in the form

$$
x(t)=\left(\frac{a(t)}{I_{3}}\right)^{\frac{1}{n}} e^{\frac{-1}{n} \int_{0}^{t} \gamma\left(t^{\prime}\right) d t^{\prime}}\left[C+\frac{m}{n} \int_{0}^{t}\left(\beta\left(t^{\prime}\right)\left(\frac{a\left(t^{\prime}\right)}{I_{3}}\right)^{\frac{m}{n}} e^{\frac{-m}{n} \int_{0}^{t^{\prime}} \gamma\left(t^{\prime \prime}\right) d t^{\prime \prime}}\right) d t^{\prime}\right]^{\frac{-1}{m}} .
$$

We note that the general solution (41) now contains only three arbitrary constants, namely, $\hat{I}_{1}, \hat{I}_{2}$ and $C$.

Since we have three arbitrary parameters in the linear ODE (35), one can consider certain specific sub-cases of physical interest. In the following, we discuss some of them.

\subsection{Case (i) $c_{i}=0, i=1,2,3$}

In this case, from equation (35), we have a simple linear equation $\frac{d^{3} U}{d t^{3}}=0$ which can be connected to the third order nonlinear ODE (36) (with $c_{1}, c_{2}, c_{3}=0$ ) through the nonlocal transformation (12). The general solution of (36) can be fixed easily from (41) in the form

$$
\begin{aligned}
x(t)=\left(\frac{1}{2} t^{2}+\right. & \left.I_{1} t+I_{2}\right)^{\frac{1}{n}} e^{\frac{-1}{n} \int_{0}^{t} \gamma\left(t^{\prime}\right) d t^{\prime}} \\
& \times\left[C+\frac{m}{n} \int_{0}^{t}\left(\beta\left(t^{\prime}\right)\left(\frac{1}{2} t^{\prime 2}+I_{1} t^{\prime}+I_{2}\right)^{\frac{m}{n}} e^{-\frac{m}{n} \int_{0}^{t^{\prime}} \gamma\left(t^{\prime \prime}\right) d t^{\prime \prime}}\right) d t^{\prime}\right]^{\frac{-1}{m}},
\end{aligned}
$$

where $I_{1}, I_{2}$ and $C$ are arbitrary constants.

Equation (36) includes a class of important nonlinear systems. For example, choosing $n=1, m=1, \gamma(t)=0$ and $\beta(t)=k$ equation (36) becomes

$$
\dddot{x}+4 k x \ddot{x}+3 k \dot{x}^{2}+6 k^{2} x^{2} \dot{x}+k^{3} x^{4}=0 .
$$

Equation (43) is a special case of the Chazy equation XII (with $N=2$ and parametric restrictions $A=0, B=0$ in Ref. [18]), which has been studied in detail in Refs. [16-22]. The general solution can be fixed easily from (42) in the form

$$
x(t)=\left(\frac{\frac{k t^{2}}{2}+I_{1} t+I_{1} I_{2}}{\frac{k^{2} t^{3}}{6}+\frac{k I_{1}}{2} t^{2}+k I_{1} I_{2} t+I_{1} I_{3}}\right) .
$$


3.2. Case (ii) $c_{3}=0, c_{2}=0$ and $c_{1}=$ constant

In this case, the linear equation (35) becomes $\ddot{U}+c_{1} \ddot{U}=0$ and the nonlocal transformation (12) transforms this ODE to the form (36) with $c_{2}, c_{3}=0$. In this case we have the general solution of the form

$$
\begin{aligned}
x(t)=\left(e^{-c_{1} t}\right. & \left.+I_{1} t+I_{2}\right)^{\frac{1}{n}} e^{\frac{-1}{n} \int_{0}^{t} \gamma\left(t^{\prime}\right) d t^{\prime}} \\
& \times\left[C+\frac{m}{n} \int_{0}^{t}\left(\beta\left(t^{\prime}\right)\left(e^{-c_{1} t^{\prime}}+I_{1} t^{\prime}+I_{2}\right)^{\frac{m}{n}} e^{-\frac{m}{n} \int_{0}^{t^{\prime}} \gamma\left(t^{\prime \prime}\right) d t^{\prime \prime}}\right) d t^{\prime}\right]^{\frac{-1}{m}},
\end{aligned}
$$

where $I_{1}, I_{2}$ and $C$ are arbitrary constants. Choosing $n=1, m=1, \gamma(t)=0$ and $\beta(t)=k$ equation (36) becomes

$$
\dddot{x}+\left(c_{1}+4 k x\right) \ddot{x}+3 k \dot{x}^{2}+3 k\left(c_{1}+2 k x\right) x \dot{x}+\left(c_{1}+k x\right) k^{2} x^{3}=0 .
$$

The general solution of equation (46) can be fixed easily from (45) in the form

$$
x(t)=\left(\frac{e^{-c_{1} t}+I_{1} t+I_{2}}{C+\frac{k}{2 c_{1}}\left(2 e^{-c_{1} t}+c_{1} I_{1} t^{2}+2 c_{1} I_{2} t\right)}\right) .
$$

\subsection{Case (iii) $c_{1}=0, c_{2}=0$ and $c_{3}=$ constant}

In this case, the linear ODE (35) assumes the form $\dddot{U}+c_{3} U=0$ and the nonlocal transformation (12) transforms this equation, $\dddot{U}+c_{3} U=0$, to the nonlinear ODE (36) with $c_{1}, c_{2}=0$. The general solution of (36) turns out to be

$$
\begin{aligned}
x(t) & =\left(I_{1} e^{-k t}+e^{\frac{k}{2} t} \cos \left(\frac{\sqrt{3} k}{2} t+\delta\right)\right)^{\frac{1}{n}} e^{\frac{-1}{n} \int_{0}^{t} \gamma\left(t^{\prime}\right) d t^{\prime}} \\
& \times\left[C+\frac{m}{n} \int_{0}^{t}\left(\beta\left(t^{\prime}\right) e^{-\frac{m}{n} \int_{0}^{t^{\prime}} \gamma\left(t^{\prime \prime}\right) d t^{\prime \prime}}\left(I_{1} e^{-k t^{\prime}}+e^{\frac{k}{2} t^{\prime}} \cos \left(\frac{\sqrt{3} k}{2} t^{\prime}+\delta\right)\right)^{\frac{m}{n}}\right) d t^{\prime}\right]^{\frac{-1}{m}},
\end{aligned}
$$

where $c_{3}=k^{3}$ and $I_{1}, C$ and $\delta$ are arbitrary constants. By choosing $n=1, m=$ $1, \gamma(t)=0$ and $\beta(t)=k$ in equation (36) one can get a special case of the Chazy equation XII (with $N=2$ and parametric restrictions $A=c_{3}$ and $B=0$ in Ref. [18])

$$
\dddot{x}+4 k x \ddot{x}+3 k \dot{x}^{2}+6 k^{2} x^{2} \dot{x}+k^{3} x^{4}+c_{3} x=0 .
$$

The general solution of (49) can be fixed easily from (48) in the form

$$
x(t)=\left(\frac{I_{1} e^{-k t}+e^{\frac{k}{2} t} \cos \left(\frac{\sqrt{3} k}{2} t+\delta\right)}{C-\frac{k}{2 k}\left(2 I_{1} e^{-k t}-e^{\frac{k}{2} t}\left(\cos \left(\frac{\sqrt{3} k}{2} t+\delta\right)+\sqrt{3} \sin \left(\frac{\sqrt{3} k}{2} t+\delta\right)\right)\right.}\right) .
$$

\subsection{Case (iv) $c_{i}=$ constant, $i=1,2,3$}

In this case the nonlocal transformation transforms the nonlinear ODE (36) to the linear ODE (35). The general solution of the latter becomes

$$
\begin{aligned}
x(t)= & \left(e^{m_{1} t}+I_{1} e^{m_{2} t}+I_{2} e^{m_{3} t}\right)^{\frac{1}{n}} e^{\frac{-1}{n} \int_{0}^{t} \gamma\left(t^{\prime}\right) d t^{\prime}} \\
& \times\left[C+\frac{m}{n} \int_{0}^{t}\left(\beta\left(t^{\prime}\right)\left(e^{m_{1} t^{\prime}}+I_{1} e^{m_{2} t^{\prime}}+I_{2} e^{m_{3} t^{\prime}}\right)^{\frac{m}{n}} e^{-\frac{m}{n} \int_{0}^{t^{\prime}} \gamma\left(t^{\prime \prime}\right) d t^{\prime \prime}}\right) d t^{\prime}\right]^{\frac{-1}{m}},
\end{aligned}
$$


where $I_{1}, I_{2}$ and $C$ are arbitrary constants and $m_{i}^{3}+c_{1} m_{i}^{2}+c_{2} m_{i}+c_{3}=0, i=1,2,3$, and $m_{2,3}=\frac{1}{2}\left[-\left(m_{1}+c_{1}\right) \pm \sqrt{-3 m_{1}^{2}-2 c_{1} m_{1}+c_{1}^{2}-4 c_{2}}\right]$.

Equation (36) includes a class of important nonlinear systems. For $n=1, m=$ $1, \gamma(t)=0$ and $\beta(t)=k$ equation (36) becomes

$$
\begin{aligned}
\dddot{x}+\left(c_{1}+4 k x\right) \ddot{x}+3 k \dot{x}^{2} & +\left(c_{2}+3 k c_{1}+6 k^{2} x\right) x \dot{x} \\
& +\left(c_{1}+k x\right) k^{2} x^{3}+c_{2} k x^{2}+c_{3} x=0 .
\end{aligned}
$$

Equation (52) is a generalized version of the above special cases (43) and (49) of the Chazy equation XII, with additional terms. The general solution of (52) can be fixed easily from (51) in the form

$$
x(t)=\left(e^{m_{1} t}+I_{1} e^{m_{2} t}+I_{2} e^{m_{3} t}\right)\left[C+k\left(\frac{e^{m_{1} t}}{m_{1}}+\frac{I_{1}}{m_{2}} e^{m_{2} t}+\frac{I_{2}}{m_{3}} e^{m_{3} t}\right)\right]^{-1} .
$$

To our knowledge, the above solution is a new one. For a detailed description of Chazy class of third order ordinary differential equations, their analytic properties and their general solutions one may refer to Ref. [18], besides the original work of Chazy [16].

In this section, we demonstrated the nonlocal connection that exists between linear and nonlinear ODEs of third order. In the following section, we extend the theory to $n$th order ODEs.

\section{4. $n$th order equations}

Let us consider a most general linear ODE of the form

$$
\left(\frac{d^{n}}{d t^{n}}+c_{1} \frac{d^{(n-1)}}{d t^{(n-1)}}+\ldots+c_{n-1} \frac{d}{d t}+c_{n}\right) U(t)=0
$$

where $c_{i}$ 's, $i=1,2, \ldots n$, are arbitrary constants. The nonlocal transformation (12) connects (54) to the nonlinear ODE of the form

$$
\left(D_{h}^{(n)}+c_{1} D_{h}^{(n-1)}+\ldots+c_{n-1} D_{h}^{(1)}+c_{n}\right) x^{n}=0
$$

where $D_{h}^{(n)}=\left(\frac{d}{d t}+\beta(t) x^{m}+\gamma(t)\right)^{n}$. For $n=2$ and 3 equation (55) coincides with (13) and (36) respectively. Repeating the procedure described in Secs. 2 and 3 one can derive the general solution of the $n$th order nonlinear ODE, (55), in the form

$$
x(t)=e^{\frac{1}{n} \int_{0}^{t}\left(\hat{a}\left(t^{\prime}\right)-\gamma\left(t^{\prime}\right)\right) d t^{\prime}}\left[C+\frac{m}{n} \int_{0}^{t}\left(\beta\left(t^{\prime}\right) e^{\frac{m}{n} \int_{0}^{t^{\prime}}\left(\hat{a}\left(t^{\prime \prime}\right)-\gamma\left(t^{\prime \prime}\right)\right) d t^{\prime \prime}}\right) d t^{\prime}\right]^{\frac{-1}{m}} .
$$

Here again $\hat{a}(t)=\frac{\dot{a}}{a}$, where $a$ is the known solution of equation (154). The solution contains $n+1$ integration constants, namely, $I_{1}, I_{2}, \ldots I_{n}$ and $C$. However, as was done in the second and third order ODEs, respectively, we can absorb one integration constant and rewrite the solution in such a way that it contains only $n$ integration constants. Doing so we arrive at

$$
x(t)=\left(\frac{a(t)}{I_{n}}\right)^{\frac{1}{n}} e^{\frac{-1}{n} \int_{0}^{t} \gamma\left(t^{\prime}\right) d t^{\prime}}\left[C+\frac{m}{n} \int_{0}^{t}\left(\beta\left(t^{\prime}\right)\left(\frac{a\left(t^{\prime}\right)}{I_{n}}\right)^{\frac{m}{n}} e^{\frac{-m}{n} \int_{0}^{t^{\prime}} \gamma\left(t^{\prime \prime}\right) d t^{\prime \prime}}\right) d t^{\prime}\right]^{\frac{-1}{m}} .
$$


By appropriately fixing the constants and arbitrary functions in equation (55) one can deduce the nonlinear equation of interest. The general solution can also be fixed unambiguously from equation (157).

\section{General Theory}

So far we focussed our attention only on the cases in which the nonlocal transformation that connectes the nonlinear ODEs with constant coefficient linear ODEs. However, one can also explore the hidden connection between nonlinear ODEs and variable coefficient linear ODEs. In the following we briefly discuss the underlying theory.

Let us begin with a $n$th order linear ODE of the form

$$
\left(\frac{d^{n}}{d t^{n}}+c_{1}(t) \frac{d^{(n-1)}}{d t^{(n-1)}}+\ldots+c_{n-1}(t) \frac{d}{d t}+c_{n}(t)\right) U(t)=0
$$

where $c_{i}$ 's, $i=1,2, \ldots n$, are functions of $t$, whose general solution be $U=a(t)$, where $a(t)$ is some known function. Now we consider a general nonlocal transformation of the form

$$
U(t)=g(t, x) e^{\int_{0}^{t} f\left(t^{\prime}, x\right) d t^{\prime}},
$$

where $f(t, x)$ and $g(t, x)$ are arbitrary functions of $t$ and $x$, and substitute it into (58) so that the latter becomes a nonlinear ODE of the form

$$
\left(D_{h}^{(n)}+c_{1}(t) D_{h}^{(n-1)}+\ldots+c_{n-1}(t) D_{h}^{(1)}+c_{n}(t)\right) g(t, x)=0,
$$

where $D_{h}^{(n)}=\left(\frac{d}{d t}+f(t, x)\right)^{n}$. Since we have assumed that the multiplicative function $g(t, x)$ is a function of the variables $t$ and $x$, the associated nonlinear ODE, (60), takes a very complicated form. However, the task is to deduce the interesting cases from (60) which can be integrated explicitly. Again using the identity

$$
\frac{\dot{U}}{U}=\frac{g_{x} \dot{x}+g_{t}}{g}+f,
$$

with $U=a(t)$, equation (61) can be brought to the form

$$
\dot{x}=(\hat{a}(t)-f(t, x)) \frac{g(t, x)}{g_{x}(t, x)}-\frac{g_{t}(t, x)}{g_{x}(t, x)}, \quad \hat{a}=\frac{\dot{a}}{a} .
$$

Now, it is well known that only for certain specific forms of $f$ and $g$, equation (62) can be integrated [23]. At least for these cases, the general solution for the nonlinear ODE (601) can be obtained explicity. In this way one can classify a class of integrable nonlinear ODEs of any order through nonlocal transformations of the form (59).

We note that the equation (60) can also be obtained from equation (58) by using a simple "gauge transformation"

$$
\frac{d^{n}}{d t^{n}} \rightarrow D_{h}^{(n)}=\left(\frac{d}{d t}+f(t, x)\right)^{n}, \quad f(t, x)=\frac{d}{d t} h(t, x)
$$

and

$$
U(t) \rightarrow g(x, t)
$$


Finally, we mention that in the case $f(t, x)=0$ the nonlocal transformation, (59), becomes purely a local one and the resultant linearizable equations can be directly obtained from equation (60).

\section{Conclusion}

In this paper, we have developed a novel way of identifying integrable nonlinear ODEs by connecting linear and nonlinear oscillator equations of any order through suitable nonlocal transformations. The proposed method is simple and straightforward. More importantly, our procedure not only identifies a class of integrable nonlinear ODEs of any order but also unambiguously gives their underlying solutions and thereby leads to the complete understanding of the dynamics of the given nonlinear system. As we have pointed out in the introduction, the modified Emden type equation (11) admits certain unusual nonlinear dynamical properties. The dynamical properties underlying other integrable nonlinear ODEs still remain to be explored in detail and are possibly worth investigating further. It is also of interest to investigate the existence of the above types of nonlocal connections in the case of partial differential equations as well, which we hope to pursue further.

\section{Acknowledgments}

The work of VKC is supported by Council of Scientific and Industrial Research in the form of a Senior Research Fellowship. The work of ML forms part of the Department of Science and Technology, Government of India, research project.

\section{References}

[1] Chandrasekar V K, Senthilvelan M and Lakshmanan M 2005 Phys. Rev. E72 066203; A nonlinear oscillator with unusual dynamical properties in Proceedings of the Third National Conference on Nonlinear Systems and Dynamics, Allied publishers, Chennai (2006) p. 1-4

[2] Carinena J F, Ranada M F and Santander M 2005 J. Math. Phys. 46 062703; arxiv:math-ph/0505028

[3] Ince E L 1956 Ordinary Differential Equations (New York:Dover)

[4] Mahomed F M and Leach P G L 1985 Quaestiones Math. 8 241; Mahomed F M and Leach P G L 1989 Quaestiones Math. 12121

[5] Leach P G L 1985 J. Math. Phys. 26 2510; Leach P G L, Feix M R and Bouquet S 1988 J. Phys. A: Math. Gen. 29 2563; Lemmer R L and Leach P G L 1993 J. Phys. A: Math. Gen. 265017

[6] Feix M R, Geronimi C, Cairo L, Leach P G L, Lemmer R L and Bouquet S 1997 J. Phys. A: Math. Gen. 307437

[7] Dixon J M and Tuszynski J A 1990 Phys. Rev. A 414166

[8] Chandrasekar V K, Senthilvelan M and Lakshmanan M 2005 Proc. R. Soc. London A 4612451

[9] Chandrasekar V K, Senthilvelan M and Lakshmanan M 2006 J. Math. Phys. 47023508

[10] Chandrasekar V K, Senthilvelan M and Lakshmanan M 2006 J. Phys. A: Math. Gen. 39 L69

[11] Smith R A 1961 J. London Math. Soc. 36, 33

[12] Sawada K and Osawa T 1978 J. Phys. Soc. Japan 441730

[13] Gonzalez D L and Piro O 1983 Phys. Rev. Lett. 50 870; Phys. Rev. A 302788 
[14] Pinney E 1950 Proc. Am. Math. Soc 1681

[15] Lewis H R Jr 1967 Phys. Rev. Lett. 18 510; 1968 J. Math. Phys. 91976

[16] Chazy J 1911 Acta Math. 34317

[17] Halburd R 1999 Nonlinearity 12931

[18] Cosgrove C M 2000 Stud. in App. Math. 104 1; 104171

[19] Mugan U and Jrad F 2002 J. Nonlinear Math. Phys. 9282

[20] Euler N and Euler M 2004 J. Nonlinear Math. Phys. 11399

[21] Euler M, Euler N and Leach P G L 2005/2006 The Riccati and Ermakov-Pinney hierarchies, Report No. 08, Institut Mittag-Leffler, Sweden

[22] Chandrasekar V K, Senthilvelan M and Lakshmanan M 2006 Proc. R. Soc. London A 462 (In Press)

[23] Murphy G M 1969 Ordinary Differential Equations and Their Solutions (Affiliated East-west Press, New Delhi) 